Rogério Gomes dos Reis GUIDON

SérGio Floriano de TOleDO²

MAURÍCIO SAITO

RODRIGO FERREIRA BUZZINI $\left.\right|^{3}$

Cesar Cilento Pontes ${ }^{4}$

EDUARDO DE SOUZA ${ }^{5}$

LUIZ CAMANO

Artigos originais

Palavras-chave

Cesárea

Parto

Técnica de sutura Modelos animais

Keywords

Cesarean section

Parturition

Suture techniques

Models anima

Correspondência:

Rogério Gomes dos Reis Guidon Avenida Onze de Junho, 970, apto. 31 CEP 04041-003 - São Paulo/SP Fones: 3884-5191/9933-3091

Recebido

03/09/2007

Aceito com modificacões 20/12/2007

\title{
Avaliação anatomopatológica de cicatrizes uterinas de acordo com o tipo de sutura cirúrgica (modelo experimental)
}

\author{
Anatomopathological evaluation of uterine scars according \\ to the type of surgical suture (experimental model)
}

\section{Resumo}

OBJETIVO: comparar, macro e microscopicamente, cicatrizes uterinas pós-cesáreas, nas quais foram feitas suturas com pontos separados, contínuos e contínuos ancorados. MÉTODOS: utilizamos três coelhas prenhes, realizando parto cesáreo no $26^{\circ}$ dia de prenhez, com três incisões em cada corno uterino. As histerorrafias foram realizadas com fio Vicry ${ }^{\circledR} 00$, com suturas distintas (pontos separados, sutura contínua e contínua ancorada). № $60^{\circ}$ dia pós-parto, realizamos histerectomia total abdominal e anexectomia bilateral, para avaliação das cicatrizes cirúrgicas. Na macroscopia, avaliamos o grau de retração cicatricial (longitudinal e transversal), o depósito de fibrina, presença de aderências e integridade dos fios de sutura. Na microscopia, utilizamos coloração de hematoxilina-eosina, para contagem de vasos sangüíneos e fibroblastos, e a coloração do tricômio de Masson, para quantificação do colágeno. Para a análise comparativa das cicatrizes, utilizamos os testes de Friedman e exato de Fisher, adotando nível de significância de 5\%. RESULTADOS: foram obtidas 18 cicatrizes, seis para cada tipo de sutura. Obtivemos as seguintes médias $0,5 / 0,4 / 0,5$ ( $p=0,069)$ para os graus de retração longitudinal e 0,3/0,4/0,3 (p=0,143) para os graus de retração transversal, respectivamente para as suturas com pontos separados, contínuo e contínuo ancorado. Todas as suturas apresentaram depósito de fibrina regular, ausência de aderência e reabsorção integral dos fios. Na microscopia, apuramos a média de vasos sangǘneos $(158,5 / 139,3 / 172,1$; $p=0,293)$, de fibroblastos $(351,6 / 345,8 / 354,3 ; p=0,311)$ e da porcentagem de tecido colágeno $(44,0 / 45,5 / 48,5$; $\mathrm{p}=0,422$ ), respectivamente para as suturas com pontos separados, contínuo e contínuo ancorado. CONCLUSÕES: a técnica de histerorrafia na cesárea de coelhas (pontos simples, sutura contínua e contínua ancorada) não determinou diferenças estatísticas significantes em relação aos parâmetros macroscópicos e microscópicos avaliados.

\section{Abstract}

PURPOSE: to compare macro and microscopically, surgical uterine sutures in female rabbits, after caesarean section utilizing separate, continuous and continuous anchored suture stitches. METHODS: three New Zealand female rabbits in their first pregnancy were used. The caesarean section was carried out at the $26^{\text {th }}$ day of gestation and three incisions were performed in each uterus. The hysterorrhaphy was performed with a 00 Vicry ${ }^{\circledR}$ thread, and a different suture technique was employed for each incision. Total hysterectomy and adnexectomy were done at the $60^{\text {th }}$ day post-delivery with the preservation of eventual adhesions for the evaluation of the surgical scars. The extent of scar retraction, amount of fibrin deposit and the suture integrity were evaluated through macroscopy. For the evaluation through microscopy, hematoxylin eosin technique was used for cellular colorimetry, and Masson's trichrom to evidence collagen. The statistical non-parametric Friedman's test was employed for the matching hypothesis, and Fisher's exact test to verify the homogeneity of the techniques (level of significance: $5 \%$ ). RESULTS: a total of 18 scars were obtained (six scars per suture). The following mean values were obtained for the longitudinal $(0.5 / 0.4 / 0.5, p=0.069$ ) and transversal retraction degrees $(0.3 / 0.4 / 0.3, p=0.143)$ respectively for separate, continuous and continuous anchored suture techniques. All sutures presented regular fibrin deposit, no adhesions and integral absorption of the stitches. The mean value of the blood vessels (158.5/139.3/172.1; $p=0.293$ ), fibroblasts (35 1.6/345.8/354.3; $p=0.311$ ) and of collagen percentage (44.0/45.5/48.5; $p=0.422$ ) were calculated through microscopy, respectively for separate, continuous and continuous anchored suture techniques. CONCLUSIONS: the type of hysterorrhaphy technique of caesarean section in female rabbits did not generate any significant statistical difference in the macroscopic and microscopic parameters evaluated.

Trabalho realizado na Faculdade de Ciências Médicas de Santos do Centro Universitário Lusíada - FCMS/Unilus - Santos (SP), Brasil. Escola Paulista de Medicina da Universidade Federal de São Paulo - UNIFESP - São Paulo (SP), Brasil.

Coordenador da Disciplina de Obstetrícia da Faculdade de Ciências Médicas de Santos do Centro Universitário Lusíada - FCMS/Unilus - Santos (SP), Brasil.

2 Professor de Obstetrícia da Faculdade de Ciências Médicas de Santos do Centro Universitário Lusíada - FCMS/Unilus - Santos (SP), Brasil. ${ }^{3}$ Residente de Ginecologia e Obstetrícia do Hospital do Servidor Público Estadual; Ex-aluno Faculdade de Ciências Médicas de Santos do Centro Universitário Lusíada - FCMS/Unilus - Santos (SP), Brasil.

${ }^{4}$ Professor de Fisiopatologia da Faculdade de Ciências Médicas de Santos do Centro Universitário Lusíada - FCMS/Unilus - Santos (SP), Brasil. Professor-Associado do Departamento de Obstetrícia da Escola Paulista de Medicina da Universidade Federal de São Paulo - UNIFESP- São Paulo (SP), Brasil.

- Professor Titular do Departamento de Obstetrícia da Escola Paulista de Medicina da Universidade Federal de São Paulo - UNIFESP - São Paulo (SP), Brasil. 


\section{Introdução}

A adequada cicatrização da parede uterina obtida após parto operatório é de suma importância, uma vez que ela determinará a via do futuro parto e, em alguns casos, o próprio porvir obstétrico. A ruptura da cicatriz uterina representa risco de vida fetal e materno. Quando ocorre uma ruptura uterina extensa, a intervenção imediata é necessária e, por vezes, a histerectomia é a única opção cirúrgica. O feto, nesta situação, muitas vezes sucumbe ou cursa com seqüelas graves no seu desenvolvimento neuro-psíquico-motor ${ }^{1}$. A ocorrência de ruptura depende do tipo e localização da sutura uterina prévia, sendo que a literatura aponta para a seguinte variação: incisão clássica (4 a 9\%); incisão em T (4 a 9\%); incisão segmento-corporal (1 a 7\%); incisão segmentar transversa $(0,2 \text { a } 1,5 \%)^{2,3}$.

A resistência das cicatrizes uterinas está diretamente subordinada ao número de fibras seccionadas e, naturalmente, à técnica de histerorrafia utilizada. Todo músculo cortado une-se por meio de um processo cicatricial que envolve depósito de tecido conjuntivo, constituindo área de menor resistência, capaz de ceder em face de tração enérgica ${ }^{4}$.

Embora a cesariana seja o procedimento cirúrgico mais realizado em todo o mundo, as técnicas empregadas para a sua realização ainda são motivos de discussão e estudo, principalmente no que se refere ao fechamento cirúrgico do útero, não existindo ainda uma normatização para sua realização.

A não uniformidade de técnicas cirúrgicas para realização de cesarianas motivou, em fevereiro de 2004, o início de uma metanálise no Instituto Cochrane, o qual ainda não define a melhor normatização quanto à técnica cirúrgica para histerorrafias.

Algumas avaliações clínicas relacionando tempo cirúrgico, custos e aumento de morbidade em função das suturas uterinas contínua e separada já foram realizadas. A sutura que apresentou redução do tempo cirúrgico, redução de gasto de fio cirúrgico e menor uso de analgésico no puerpério foi a contínua em um único plano ${ }^{6}$.

Estudos experimentais envolvendo análise anatomopatológica e avaliação do emprego ou não das suturas uterinas já foram realizados. Surpreendentemente, evidenciou-se, em úteros não suturados, menor reação local com reduzida fase de fibroplasia e diminuição da necrose local $^{7}$.

A literatura carece de estudo experimental que tenha avaliado, à luz da anatomia patológica, os tipos de sutura uterina mais freqüentemente realizados nos serviços de obstetrícia, durante a realização das cesáreas.

\section{Métodos}

Realizou-se estudo experimental, para o qual foram selecionadas três coelhas (Oritolagus cuniculus), linhagem Nova Zelândia, fêmeas, adultas, procedentes do Biotério da Faculdade de Ciências Médicas de Santos do Centro Universitário Lusíada (Unilus). Todas as coelhas estavam na primeira prenhez e o dia inicial de gestação foi determinado pela presença de espermatozóides no fórnice vaginal no dia do acasalamento. Foram mantidas no biotério da instituição, acondicionadas em condições padrão para a realização da pesquisa.

Este trabalho foi aprovado pelo Comitê de Ética em Pesquisa da Escola Paulista de Medicina da Universidade Federal de São Paulo (Unifesp/EPM).

As coelhas foram submetidas a duas intervenções cirúrgicas no centro cirúrgico do biotério, seguindo-se os preceitos de assepsia e anti-sepsia. A antibioticoterapia profilática foi praticada, nessas intervenções, uma hora antes e seis horas após o procedimento; foi administrada ampicilina ( $250 \mathrm{mg} /$ dose). A prática anestésica incluiu utilização de xylasina ( $5 \mathrm{mg} / \mathrm{kg}$ ) e ketamina $(25 \mathrm{mg} / \mathrm{kg}$ ), por via intramuscular. A equipe cirúrgica foi sempre a mesma em todas as operações. Foi considerado critério de exclusão do estudo a evolução pós- cirúrgica não fisiológica, atestada pelo seguimento clínico do animal.

Na primeira cirurgia, para realização do parto cesáreo, no $26^{\circ}$ dia de prenhez, realizou-se laparotomia longitudinal mediana feita com bisturi frio lâmina $\mathrm{n}^{\circ} 10$, da pele ao peritônio parietal; procedeu-se à exteriorização do útero gravídico da cavidade abdominal e foram feitas três histerotomias em cada corno uterino (direito e esquerdo) a bisturi frio na região corporal anterior, transversalmente ao sentido dos cornos, no mesmo sentido das fibras circulares uterinas e da vascularização uterina, resultando em menor dano tecidual. Cada incisão teve aproximadamente $3 \mathrm{~cm}$ de extensão e distou aproximadamente 3 cm da outra incisão (mensuração realizada com auxílio de histerômetro), conforme a Figura 1. A retirada das crias e anexos placentários foi feita por ordenha suave. Em todas as suturas, utilizou-se fio de Vicryl ${ }^{\circledR} 00$. As incisões foram suturadas de três formas distintas: com pontos separados, contínuos e contínuos ancorados (com uma laçada do fio no ponto anterior, travando o fio de sutura cirúrgica), proporcionando um tipo diferente de sutura a cada corno uterino. Os pontos distaram aproximadamente 0,5 centímetro, entre si, totalizando, em média, seis pontos por incisão. As suturas tiveram apenas tensão de aproximação, para evitar isquemia acentuada, e os nós foram dados com auxílio de porta-agulha; os pontos abrangeram o miométrio, sendo poupado o endométrio. A revisão da hemostasia foi rigorosa, com passagem de pontos separados para coibir 
eventuais vasos sangrantes, se necessário. Os planos da parede abdominal foram suturados com fio Vicryl 00, com pontos separados; a pele recebeu sutura com pontos separados de náilon 00 .

Foi colocada veste justa, protegendo a incisão cirúrgica, e a coelha foi encaminhada para gaiola, devidamente identificada em sua orelha, com números romanos de I a III.

A segunda cirurgia realizada nas coelhas (histerectomia, com retirada completa de corpo e colo uterino, além de anexos, e preservando eventuais aderências com peritônio, epíplon ou órgãos vizinhos) ocorreu no $60^{\circ}$ dia pós-parto, para realização do estudo comparativo das suturas uterinas. As peças cirúrgicas foram avaliadas macroscópica e microscopicamente. $\mathrm{O}$ patologista não tinha conhecimento prévio da localização dos tipos de sutura.

A avaliação macroscópica foi realizada 24 horas após a histerectomia, no momento do preparo das peças para a microscopia, compreendendo: avaliação do grau de retração cicatricial (extensão da sutura no sentido longitudinal versus transverso, utilizando-se régua milimetrada), conforme a Figura 2, depósito de fibrina (regular versus irregular), aderências do útero com órgãos vizinhos (presente versus ausente) e integridade dos fios de sutura (íntegros versus absorvidos).

Para avaliação microscópica, as peças cirúrgicas foram fixadas em solução de formol a 10\% tamponada, durante um período de 24 horas; as áreas de sutura foram submetidas a cortes seqüenciais transversais à linha de incisão em toda a sua extensão; os fragmentos teciduais foram processados em banhos seqüenciais de álcoois a $80 \%, 90 \%$ e álcool absoluto, banho em Xylol e banho de parafina com posterior inclusão em blocos de parafina; cortes histológicos de $4 \mathrm{\eta m}$ de espessura foram obtidos por meio de micrótomo rotativo; em seguida, procedeu-se ao preparo padrão para coloração de hematoxilina-eosina e tricrômio de Masson.

A avaliação microscópica compreendeu a análise quantitativa de vasos sangüíneos, fibroblastos e do depósito de colágeno. Os primeiros foram estudados por meio da avaliação da proliferação vascular com a contagem de vasos capilares em dez campos de 100 aumentos (x) (densidade capilar por $\mathrm{mm}^{2}$ ). Foram considerados os capilares com hemácias no seu interior, utilizando-se o programa para contagem Imagetoll. A quantificação dos fibroblastos foi realizada em dez campos de aumento de 400x , com a visualização de células fusiformes com núcleo ovalado, utilizando-se o programa de contagem Imagetoll (Figura 3). A quantificação do colágeno foi feita pela técnica de representação de imagem, efetuando-se a sua quantificação pelo sistema computadorizado, constituído por microscópio (Carl Zeiss) com objetivas de 10 e

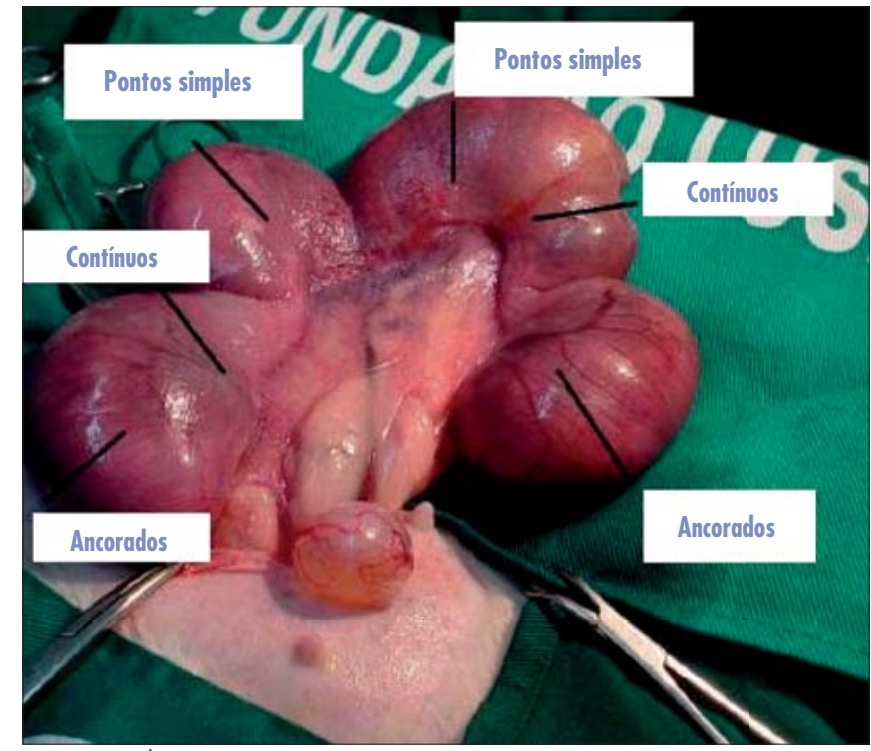

Figura 1 - Útero gravídico de coelha exteriorizado da cavidade abdominal no $26^{\circ}$ dia de prenhez. São assinaladas as áreas das incisões cirúrgicas e o tipo de sutura empregada na histerorrafia.

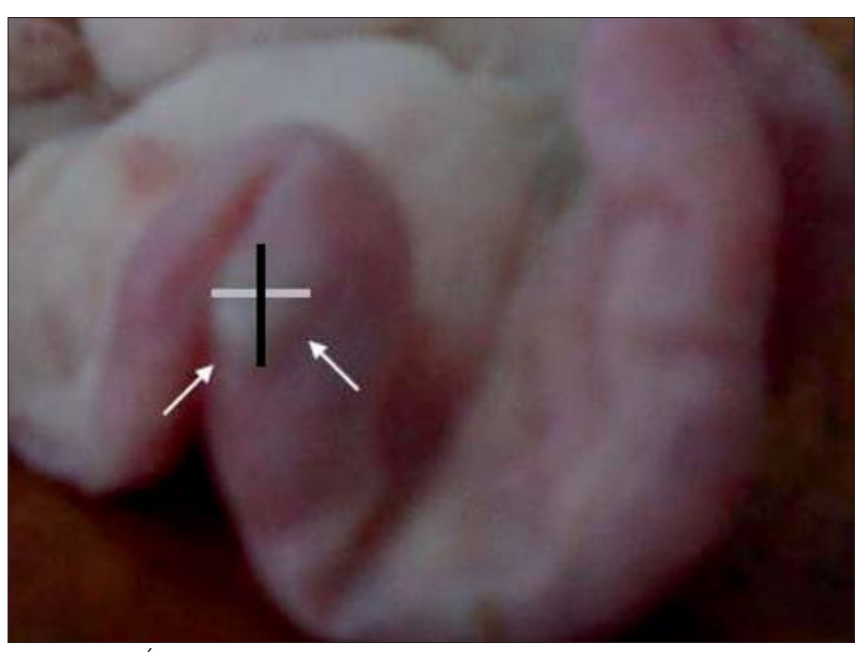

Figura 2 - Útero de coelha no $60^{\circ}$ dia pós-parto, apresentando cicatriz cirúrgica (setas). A linha branca mostra a mensuração transversal da cicatriz. A linha preta mostra a mensuração longitudinal da cicatriz uterina.

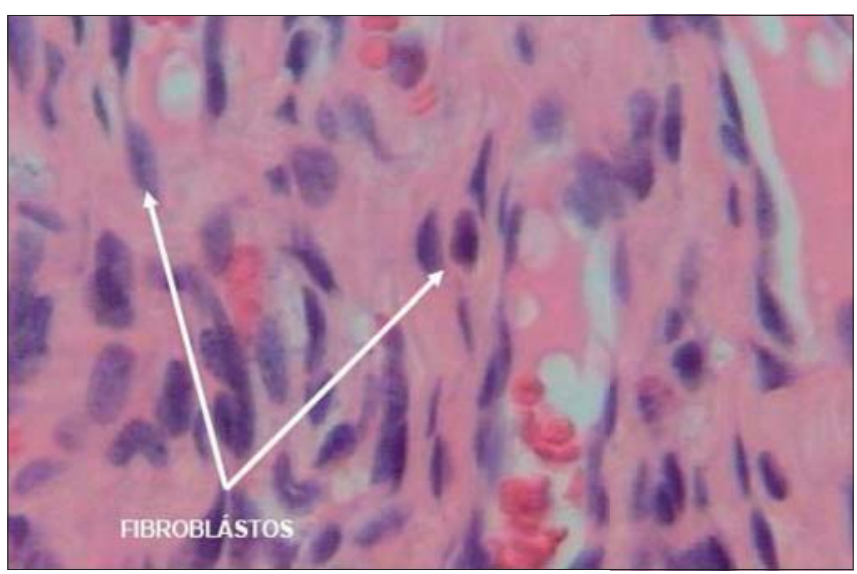

Figura 3 - Corte histológico de cicatriz uterina de coelha no $60^{\circ}$ dia pósparto cesáreo, corada com hematoxilina-eosina com aumento de 400X As setas indicam os fibroblastos. 


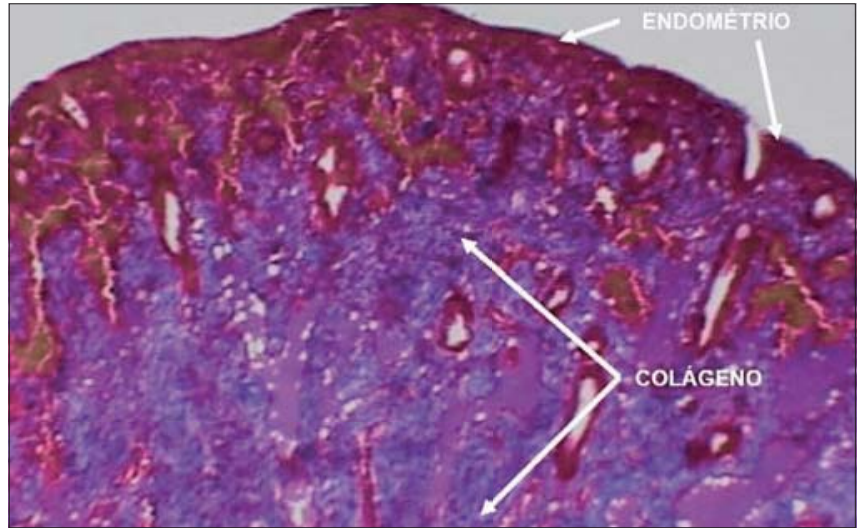

Figura 4 - Corte histológico de cicatriz uterina de coelha no $60^{\circ}$ dia póscesariana, corado com tricômio de Masson com aumento de 100X. As setas indicam endométrio e colágeno da área suturada.

40X, câmera de vídeo colorida (Sony Hyper Had), computador Pentium $200 \mathrm{mHz}$, placa gráfica para aquisição de imagem 640 x 480 pixels e 24 bits (16 milhões de cores) e software de processamento de análise de imagem Imagelab (Softium Informática Ltda.). Foram padronizadas a objetiva, a intensidade de luz do microscópio e a altura do condensador.

De cada lâmina, foram selecionados aleatoriamente três campos a um aumento de 400X. As imagens foram digitalizadas e as áreas de colágeno identificadas no programa Imagelab, usando-se distribuição de cor como parâmetro discriminante. $\mathrm{O}$ intervalo de cor foi definido de forma empírica. Por meio de tentativa e erro, uma faixa de cor foi sendo ajustada, até separar a área ocupada pelo colágeno (cor azul) em cada campo. A área separada (cor azul) representa a quantidade de colágeno existente em cada campo, sendo mostrada em porcentagem. Esse mesmo processo foi repetido em todas as lâminas (Figura 4).

Para as variáveis quantitativas (grau de retração cicatricial, número de vasos sangüíneos, fibroblastos e porcentagem de colágeno) foi feita análise pela observação dos valores mínimos e máximos e do cálculo de médias e desvios padrão e medianas. Para as variáveis qualitativas (depósito de fibrina regular ou irregular, presença de aderências e reabsorção dos fios), foram calculadas as freqüências absolutas e relativas. Para a análise da hipótese de nulidade entre as três técnicas, utilizou-se o teste não paramétrico de Friedman e, para se testar a homogeneidade das técnicas em relação às proporções, foi utilizado o teste exato de Fisher. O nível de significância utilizado para os testes foi de $5 \%$.

\section{Resultados}

Foram obtidas 18 cicatrizes, seis para cada tipo de sutura, sendo seis cicatrizes em cada coelha. Obtivemos as médias $0,5 / 0,4 / 0,5(\mathrm{p}=0,069)$ para os graus de retração
Tabela 1 - Valores de média do grau de retração longitudinal e transversal de cicatrizes uterinas de coelhas no $60^{\circ}$ dia pós-parto cirúrgico, segundo as técnicas utilizadas.

\begin{tabular}{lccccc}
\hline \multirow{2}{*}{$\begin{array}{l}\text { Técnicas de sưura } \\
\text { (pontos) }\end{array}$} & \multicolumn{2}{c}{ Retração longíitudinal } & \multicolumn{2}{c}{ Retração transversal } \\
\cline { 2 - 6 } & & Média & $\mathbf{p}^{*}$ & Média & $\mathbf{p}^{*}$ \\
\hline Simples & 6 & 0,53 & & 0,30 & \\
Contínuos & 6 & 0,47 & 0,069 & 0,40 & 0,143 \\
Ancorados & 6 & 0,53 & & 0,37 & \\
\hline
\end{tabular}

*Nível descritivo de probabilidade do teste não paramétrico de Friedman.

Tabela 2 - Valores de média do número de vasos sanguíneos, de fibroblastos e do depósito de colágeno de cicatrizes uterinas de coelhas no $60^{\circ}$ dia pós-parto cirúrgico, segundo as técnicas utilizadas.

\begin{tabular}{|c|c|c|c|c|c|c|c|}
\hline \multirow{2}{*}{$\begin{array}{l}\text { Técnicas de sutura } \\
\text { (pontos) }\end{array}$} & & \multicolumn{2}{|c|}{$\begin{array}{c}\text { Média vasos } \\
\text { sanguíneos }\end{array}$} & \multicolumn{2}{|c|}{$\begin{array}{c}\text { Média } \\
\text { fibroblastos }\end{array}$} & \multicolumn{2}{|c|}{$\begin{array}{l}\text { Média } \\
\text { colágeno }\end{array}$} \\
\hline & & & $p^{*}$ & & $p^{*}$ & & $p^{*}$ \\
\hline Simples & 6 & 158,5 & & 351,6 & & 44,0 & \\
\hline Contínuos & 6 & 139,3 & 0,293 & 345,8 & 0,311 & 45,5 & 0,422 \\
\hline Ancorados & 6 & 172,1 & & 354,3 & & 48,5 & \\
\hline
\end{tabular}

*Nível descritivo de probabilidade do teste não paramétrico de Friedman.

longitudinal $0,3 / 0,4 / 0,3(\mathrm{p}=0,143)$ e para os graus de retração transversal, respectivamente para as suturas com pontos separados, contínuo e contínuo ancorado (Tabela 1). Quanto aos outros elementos de avaliação macroscópica, em relação ao depósito de fibrina, nas três técnicas de sutura, evidenciamos que todas as cicatrizes possuíam aspecto regular, sem depósito de fibrina. Houve ainda uniformidade em relação à presença de aderências nas três técnicas (100\% das suturas sem a presença de aderências), notando-se, também que os fios foram completamente absorvidos em todas as cicatrizes.

$\mathrm{Na}$ microscopia, apuramos a média de vasos sangüíneos $(158,5 / 139,3 / 172,1 ; p=0,293)$, de fibroblastos $(351,6 / 345,8 / 354,3 ; \mathrm{p}=0,311)$ e da porcentagem de tecido colágeno $(44,0 / 45,5 / 48,5 ; \mathrm{p}=0,422)$, respectivamente para as suturas com pontos separados, contínuo e contínuo ancorado (Tabela 2).

\section{Discussão}

A histerorrafia é o principal tempo cirúrgico na cesariana, possuindo implicação direta na determinação da futura via de parto, bem como no porvir obstétrico. Falhas técnicas no momento da histerorrafia poderão contribuir para gerar ou agravar desfechos indesejáveis, imediatos ou tardios. Não só as preocupações com sangramentos cataclísmicos devem orientar a conduta do cirurgião no momento da histerorrafia. Processos mórbidos tardios estão cada vez mais presentes na clínica ginecológica, como fístulas, deiscências, hipertrofia e hiper-retração cicatricial, sangramentos vaginais irregulares e endometriose cicatricial, exemplos de algumas intercorrências advindas de má cicatrização uterina. 
Ao estudar, em 1995, à luz da anatomia patológica, uma série de 51 úteros advindos de histerectomias com cesárea segmentar prévia, evidenciou-se: $75 \%$ apresentaram irregularidade e distorção cicatricial; $65 \%$ estavam com acúmulo de endométrio sobre a cicatriz; $16 \%$ com formação poliposa; $65 \%$ com infiltração linfocitária acentuada; $92 \%$ com presença de células gigantes com resíduo de fios de sutura; $37 \%$ com descontinuidade do endométrio. Embora as indicações cirúrgicas não tenham sido especificadas, o autor relaciona os achados com a clínica de dispareunia, metrorragia, dismenorréia e dor pélvica ${ }^{8}$.

Variações técnicas, utilizando diferentes tipos de materiais, como staples, ou de substâncias bioquimicamente ativas para modular as reações cicatriciais, tentam - até o presente momento, sem o sucesso esperado - intervir e modular o processo de cicatrização ad integrum, para que a matriz tenha sua resistência e contratilidade preservadas integralmente ${ }^{9,10}$.

Em ratas, o uso do hormônio de crescimento para modular a produção do colágeno parece apresentar resultados significativos no incremento da produção deste importante elemento de cicatrização ${ }^{11}$.

A histerorrafia em um ou dois planos, o tipo de fio de sutura e o tipo de técnica cirúrgica são pontos ainda polêmicos a respeito da abordagem uterina no parto cesárea. Não é consenso, mas prevalece a opinião de que um único plano com sutura contínua tem melhor custo-benefício $^{12}$. Em estudo realizado por Hayakawa et al. ${ }^{13}$, observou-se uma redução em $20 \%$ dos defeitos na histerorrafia avaliada por ultra-sonografia transvaginal, quando realizaram-se sutura contínua decidual e sutura com pontos separados abrangendo o miométrio.

Alguns estudos clínicos relevantes já foram realizados para avaliar estados puerperais em função das técnicas de cesárea, como tempo de internação, estados febris, anemia e ruptura uterina ${ }^{6}$. Outros pesquisadores já se preocuparam com o tema "cicatrização uterina". Trabalhos experimentais avaliando a cicatriz no puerpério, com ou sem suturas, evidenciaram surpreendentemente que o grau de necrose foi menor nos úteros não suturados, como também apresentaram menor incidência de aderências, menor quantidade de fibrose, menor gasto com fios e tempo cirúrgico.

Optamos pelo estudo experimental em função das inúmeras barreiras de ordem ética que iríamos enfrentar para a aquisição de amostra significativa da cicatriz uterina humana. A coelha possui dois cornos uterinos (didelfo), que, no termo de sua prenhez, atinge um volume capaz de receber três incisões cirúrgicas em cada um, situação adequada para realizarmos três suturas em um mesmo útero (self-pairing).
Utilizamos o $60^{\circ}$ dia para realizar a segunda cirurgia (histerectomia), em decorrência do tempo de absorção completa do fio de Vicryl ${ }^{\circledR}$, que é de aproximadamente 60 dias. A ausência do fio de sutura no momento da avaliação facilitou a observação dos critérios macroscópicos e microscópicos propostos por este estudo.

O fio de sutura utilizado foi o de Vicryl ${ }^{\circledR} 00$, com 70 $\mathrm{cm}$ de comprimento, encastoado com método de fixação da agulha atraloc com agulha cilíndrica de $1 / 2$ círculo com 2,5 cm de comprimento real, apresentando fio violeta trançado. Acreditamos que a escolha da agulha e do fio de sutura foi adequada, uma vez que a agulha cilíndrica propicia divulsão das fibras miometriais, não agravando o trauma local, além do fato de o Vicryl ${ }^{\circledR}$ ser um composto de polímeros de glicolida e lactida, o que o torna praticamente inerte ao hospedeiro.

A involução uterina puerperal seria a grande responsável por aproximar de maneira adequada as bordas miometriais, diminuindo, assim, a distância entre elas e favorecendo o processo cicatricial. É conveniente lembrar que, em seres humanos, esta redução de volume se dá no sentido fundo-colo. A sutura uterina não teria, portanto, como no tecido cutâneo, a função de sustentação e coaptação das bordas, facilitando a cicatrização do tecido sempre submetido à tensão, mas a função puramente hemostática.

Aderências anexiais, peritoneais, epiplóicas ou intestinais foram avaliadas, pois cicatrizes exsudativas ou com áreas cruentas, infecções e endometriose aumentam a probabilidade da ocorrência de aderências, que, quando presentes, poderiam funcionar como marcadoras de condições inóspitas para a adequada cicatrização miometrial. Os nossos resultados, quanto à presença dessas aderências, evidenciaram globalmente ausência desse achado nas cicatrizes estudadas, independentemente da técnica de sutura.

Durante o processo de cicatrização, na fase inflamatória aguda, encontramos ativação do sistema de coagulação, tendo-se, como produto final dessa cascata, a transformação do fibrinogênio em fibrina. Essa rede de fibrina possibilita parada do sangramento e servirá como substrato sólido para a sustentação dos fibroblastos, que, por sua vez, migram para a área lesada com função destacada na fase de fibroplasia e também para produzir o colágeno. O tecido de granulação formado tem, como um dos seus componentes fundamentais, uma rede de novos vasos sangüíneos com função de reconstituir a irrigação local.

Mais recentemente, a literatura tem destacado o papel do colágeno no processo de cicatrização, visto que ele é a única matriz extracelular filamentar e o principal elemento biológico responsável por conferir a resistência 
tênsil de um tecido. Em um estudo utilizando-se tecido de cicatriz uterina de seres humanos, foi possível relacionar a associação entre deiscência uterina e a quantidade de colágeno. Esse estudo utilizou cicatrizes com deiscência e observou aumento da quantidade de colágeno. Os autores ainda identificaram redução global da expressão do fator de crescimento beta 3 , redução do fator de crescimento conjuntivo e aumento do fator de crescimento. Estes achados respaldam a hipótese de que a deiscência uterina pode ser o resultado de uma resposta bioquímica inadequada do processo de cicatrização ${ }^{14}$. Outro autor, avaliando o colágeno oriundo da cicatriz de cesárea durante o trabalho de parto, evidenciou um número maior de rupturas uterinas nas quais não ocorreu adequada modificação do colágeno ${ }^{15}$.

Nossos resultados, não evidenciando diferenças significativas entre as diversas técnicas de sutura cirúrgicas para a histerorrafia, possibilita ao cirurgião utilizar a técnica mais apropriada para cada situação. Entendemos que a sutura contínua, por representar o tipo de sutura em que há o menor gasto de fio cirúrgico, permitindo distribuição homogênea das tensões em todo o tecido suturado, e, por ser a técnica de execução mais rápida, terá maior adequação à rotina obstétrica. A técnica ancorada teria maior adequação nos segmentos mais espessos, com presença de sangramento uterino mais intenso, por se valer da sua capacidade hemostática. Os pontos separados ficariam reservados para as intercorrências infecciosas e para as histerorrafias longitudinais uterinas.

Concluímos, portanto, com uma ilação clínica deste nosso estudo, destacando a possibilidade de certa liberdade na escolha da técnica na histerorrafia da operação cesariana, de acordo com as condições obstétricas e preferências individuais, outrossim, avaliando a relação custo-benefício.

\section{Referências}

1. Farmer RM, Kirschbaum T, Potter D, Strong TM, Medearis AL. Uterine rupture during trial of labor after previous cesarean section. Am J Obstet Gynecol. 1991;165(4 Pt 1):996-1 101.

2. Pridjian G. Labor after prior cesarean section. Clin Obstet Gynecol. 1995;35(3):445-56

3. Al Sakka M, Hamsho A, Khan L. Rupture of the pregnant uterus a 21 years review. Int J Gynaecol Obstet. 1998;63(2):105-8.

4. Rezende J. Obstetrícia. 10a ed. Rio de Janeiro: Guanabara Koogan; 2005.

5. Dodd JM, Anderson ER, Gates S. Surgical techniques involving the uterus at the time of caesarean section [protocol]. Cochrane Database Syst Rev. 2004;(2):CD004732.

6. Hohlagschwandtner M, Chalubinski K, Nather A, Husslein P, Joura EA. Continous vs interrupted sutures for single-layer closure of uterine incision at cesarean section. Arch Gynecol Obstet. 2003;268(1):26-8.

7. Gul A, Simsek Y, Ugras S, Gul T. Transverse uterine incision nonclosure versus closure: an experimental study in sheep. Acta Obstet Gynecol Scand. 2000;79(10):813-7.

8. Morris H. Surgical pathology of lower uterine segment caesarean section scar: is the scar a source of clinical symptoms? Int J Gynecol Pathol. 1995; 14(1):16-20.

9. Peacock KE, Hurst BS, Marshburn PB, Matthews ML. Effects of fibrin sealant on single-layer uterine incision closure in the New Zealand white rabbit. Fertil Steril. 2006;85 Suppl 1:1261-4.
10. Wilkinson C, Enkin MW. Absorbable staples for uterine incision at caesarean section. Cochrane Database Syst Rev. 2000;(2):CD000005.

11. Bowers D, McKenzie D, Dutta D, Wheeless CR, Cohen WR. Growth hormone treatment after cesarean delivery in rats increases the strength of the uterine scar. Am J Obstet Gynecol. 2001;185(3):614-7.

12. Enkin MW, Wilkinson C. Single versus two layer suturing for closing the uterine incision at caesarean section. Cochrane Database Syst Rev. 2000;(2):CD000192.

13. Hayakawa H, Itakura A, Mitsui T, Okada M, Suzuki M, Tamakoshi $\mathrm{K}$, et al. Methods for myometrium closure and other factors impacting effects on cesarean section scars of the uterine segment detected by the ultrasonography. Acta Obstet Gynecol Scand. 2006;85(4):429-34.

14. Pollio F, Staibano S, Mascolo M, Salvatore G, Persico F, De Falco $M$, et al. Uterine dehiscence in term pregnant patients with one previous cesarean delivery: growth factor immunoexpression and collagen content in the scarred lower uterine segment. Am J Obstet Gynecol. 2006; 194(2):527-34.

15. Buhimschi CS, Buhimschi IA, Yu C, Wang H, Sharer DJ, Diamond $M P$, et al. The effect of dystocia and previous cesarean uterine scar on the tensile properties of the lower uterine segment. Am J Obstet Gynecol. 2006;194(3):873-83. 